Acta Crystallographica Section E

Structure Reports

Online

ISSN 1600-5368

\section{(4-Ethenylphenyl)diphenylphosphine selenide}

\section{Zanele H. Phasha, Sizwe Makhoba and Alfred Muller*}

Research Center for Synthesis and Catalysis, Department of Chemistry, University of Johannesburg (APK Campus), PO Box 524, Auckland Park, Johannesburg, 2006,

South Africa

Correspondence e-mail: mullera@uj.ac.za

Received 26 July 2012; accepted 1 August 2012

Key indicators: single-crystal X-ray study; $T=100 \mathrm{~K}$; mean $\sigma(\mathrm{C}-\mathrm{C})=0.002 \AA$; $R$ factor $=0.024 ; w R$ factor $=0.063 ;$ data-to-parameter ratio $=20.9$.

In the title molecule, $\mathrm{C}_{10} \mathrm{H}_{17} \mathrm{PSe}$, the $\mathrm{P}$ atom has a distorted tetrahedral environment resulting in an effective cone angle of $165^{\circ}$. The benzene ring makes dihedral angles of $70.04(8)$ and $77.28(8)^{\circ}$ with the phenyl rings, while the dihedral angle between the phenyl rings is $62.95(8)^{\circ}$. In the crystal, molecules are linked by $\mathrm{C}-\mathrm{H} \cdots \pi$ interactions.

\section{Related literature}

For background to our investigation of the steric and electronic effects of group 15 ligands, see: Roodt et al. (2003); Muller et al. (2006, 2008). For background to cone angles, see: Bunten et al. (2002); Tolman (1977); Otto (2001).

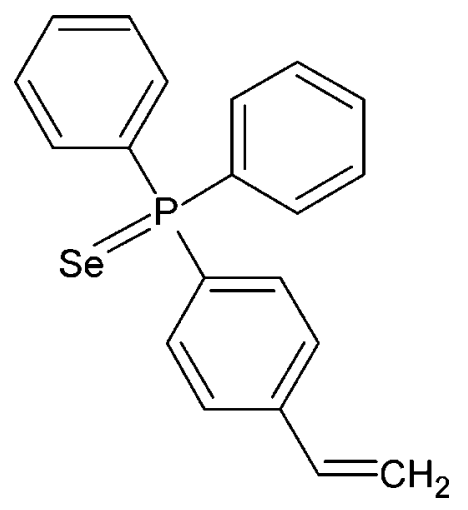

\section{Experimental}

Crystal data

$\mathrm{C}_{20} \mathrm{H}_{17} \mathrm{PSe}$

$M_{r}=367.27$

Monoclinic, $P 2_{1} / c$ $\beta=124.562(3)^{\circ}$

$V=1664.66(18) \AA^{3}$

$Z=4$

Mo $K \alpha$ radiation

Data collection

Bruker APEX DUO 4K-CCD diffractometer

Absorption correction: multi-scan (SADABS; Bruker, 2008)

$T_{\min }=0.526, T_{\max }=0.737$

Refinement

$R\left[F^{2}>2 \sigma\left(F^{2}\right)\right]=0.024$

$w R\left(F^{2}\right)=0.063$

$S=1.03$

4166 reflections

Table 1

Hydrogen-bond geometry $\left(\AA,{ }^{\circ}\right)$.

$C g 1$ and $C g 2$ are the centroids of the $\mathrm{C} 1-\mathrm{C} 6$ and $\mathrm{C} 15-\mathrm{C} 20$ rings, respectively.

\begin{tabular}{lllll}
\hline$D-\mathrm{H} \cdots A$ & $D-\mathrm{H}$ & $\mathrm{H} \cdots A$ & $D \cdots A$ & $D-\mathrm{H} \cdots A$ \\
\hline $\mathrm{C} 18-\mathrm{H} 18 \cdots C g 1^{\mathrm{i}}$ & 0.95 & 2.62 & $3.383(2)$ & 137 \\
$\mathrm{C} 3-\mathrm{H} 3 \cdots C g 2^{\mathrm{ii}}$ & 0.95 & 2.88 & $3.5889(19)$ & 133 \\
$\mathrm{C} 12-\mathrm{H} 12 \cdots C g 2^{\mathrm{iii}}$ & 0.95 & 2.85 & $3.614(2)$ & 138 \\
\hline Symmetry codes: & (i) $\quad x,-y-\frac{1}{2}, z-\frac{3}{2} ;$ & (ii) & $-x+1, y-\frac{1}{2},-z+\frac{3}{2} ;$ & (iii) \\
$x+1,-y-\frac{1}{2}, z-\frac{1}{2}$. & & & &
\end{tabular}

Data collection: APEX2 (Bruker, 2011); cell refinement: SAINT (Bruker, 2008); data reduction: SAINT and XPREP (Bruker, 2008); program(s) used to solve structure: SHELXS97 (Sheldrick, 2008); program(s) used to refine structure: SHELXL97 (Sheldrick, 2008); molecular graphics: DIAMOND (Brandenburg \& Putz, 2005); software used to prepare material for publication: Win $G X$ (Farrugia, 1999).

Research funds of the University of Johannesburg is gratefully acknowledged.

Supplementary data and figures for this paper are available from the IUCr electronic archives (Reference: IS5175).

\section{References}

Brandenburg, K. \& Putz, H. (2005). DIAMOND. Crystal Impact GbR, Bonn, Germany.

Bruker (2008). SADABS, SAINT and XPREP. BrukerAXS Inc., Madison, Wisconsin, USA.

Bruker (2011). APEX2. Bruker AXS Inc., Madison, Wisconsin, USA.

Bunten, K. A., Chen, L., Fernandez, A. L. \& Poë, A. J. (2002). Coord. Chem. Rev. 233-234, 41-51.

Farrugia, L. J. (1999). J. Appl. Cryst. 32, 837-838.

Muller, A., Meijboom, R. \& Roodt, A. (2006). J. Organomet. Chem. 691, 5794 5801.

Muller, A., Otto, S. \& Roodt, A. (2008). Dalton Trans. pp. 650-657.

Otto, S. (2001). Acta Cryst. C57, 793-795.

Roodt, A., Otto, S. \& Steyl, G. (2003). Coord. Chem. Rev. 245, 121-137.

Sheldrick, G. M. (2008). Acta Cryst. A64, 112-122.

Tolman, C. A. (1977). Chem. Rev. 77, 313-348. 


\section{supporting information}

Acta Cryst. (2012). E68, o2690 [doi:10.1107/S1600536812034289]

\section{(4-Ethenylphenyl)diphenylphosphine selenide}

\section{Zanele H. Phasha, Sizwe Makhoba and Alfred Muller}

\section{S1. Comment}

Various techniques such as crystallography, multi nuclear NMR and IR have been used to extensively study the transition metal phosphorous bond (Roodt et al., 2003). As part of this systematic investigation we have extended this study to selenium derivatives of the phosphorus ligands (see Muller et al., 2008). This way there is no steric crowding effect, albeit crystal packing effects, as normally found in transition metal complexes with bulky ligands, e.g. in

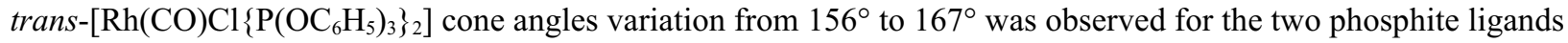
(Muller et al., 2006). The ${ }^{1} J\left({ }^{31} \mathrm{P}-{ }^{77} \mathrm{Se}\right)$ coupling can also be used as an additional probe to obtain more information regarding the nature of the phosphorous bond. Reported as part of the above continuing study, the single-crystal structure of the phosphorus containing compound, $\mathrm{SePPh}_{2}\left(4-\mathrm{C}_{2} \mathrm{H}_{3}-\mathrm{C}_{6} \mathrm{H}_{4}\right)$, where $\mathrm{Ph}=\mathrm{C}_{6} \mathrm{H}_{5}$ and 4- $\mathrm{C}_{2} \mathrm{H}_{3}-\mathrm{C}_{6} \mathrm{H}_{4}=4$-vinylbenzene, is reported here.

The title compound (Fig. 1) adopts a distorted tetrahedral arrangement about the $\mathrm{P}$ atom with average $\mathrm{C}-\mathrm{P}-\mathrm{C}$ and $\mathrm{Se}$ $-\mathrm{P}-\mathrm{C}$ angles of 105.74 and $112.98^{\circ}$, respectively. Describing the steric demand of phosphine ligands has been the topic of many studies and a variety of models have been developed (Bunten et al., 2002). Of these the Tolman cone angle (Tolman, 1977) is still the most commonly used model. Applying this model to the geometry obtained for the title compound (and adjusting the $\mathrm{Se}-\mathrm{P}$ bond distance to $2.28 \AA$ as described by Tolman), we calculated an effective cone angle from the geometry found in the crystal structure of $165^{\circ}$ (Otto, 2001). Intermolecular $\mathrm{C}-\mathrm{H} \cdots \pi$ interactions (Table 1 and Fig. 2) are observed in the crystal.

\section{S2. Experimental}

Diphenylphosphino styrene and $\mathrm{KSeCN}$ were purchased from Sigma-Aldrich and used without purification. Eqimolar amounts of $\mathrm{KSeCN}(5.8 \mathrm{mg}, 0.04 \mathrm{mmol})$ and the diphenylphosphino styrene (11.5 $\mathrm{mg}, 0.04 \mathrm{mmol})$ were dissolved in the minimum amounts of methanol $(10 \mathrm{ml})$. The $\mathrm{KSeCN}$ solution was added drop wise $(5 \mathrm{~min}$.) to the phosphine solution with stirring at room temperature. The final solution was left to evaporate slowly until dry to give crystals suitable for a single-crystal X-ray study.

Analytical data: ${ }^{1} \mathrm{H}$ NMR $\left(\mathrm{CDCl}_{3}, 400 \mathrm{MHz}\right): \delta 7.74-7.68(\mathrm{~m}, 6 \mathrm{H}), 7.45-7.42(\mathrm{~m}, 8 \mathrm{H}), 6.75-6.68(\mathrm{~m}, 1 \mathrm{H}), 5.82(\mathrm{~d}, J=$ $17.6 \mathrm{~Hz}, 1 \mathrm{H}), 5.36(\mathrm{~d}, J=4.8 \mathrm{~Hz}, 3 \mathrm{H}) ;{ }^{13} \mathrm{C}\{\mathrm{H}\} \mathrm{NMR}\left(\mathrm{CDCl}_{3}, 400 \mathrm{MHz}\right) \delta 135.8,116.7$ (ethylene), 133.0,132.9,132.7,132.6,131.6,128.6,128.5,126.3,126,2 (Ar) ${ }^{31} \mathrm{P}\{\mathrm{H}\} \mathrm{NMR}\left(\mathrm{CDCl}_{3}, 161.99 \mathrm{MHz}\right): \delta=34.71(\mathrm{t}$, $\left.{ }^{1} J\left({ }^{31} \mathrm{P}-{ }^{77} \mathrm{Se}\right)=729 \mathrm{~Hz}\right)$

\section{S3. Refinement}

All hydrogen atoms were positioned in geometrically idealized positions with $\mathrm{C}-\mathrm{H}=0.95 \AA$ (aromatic and methylene), and allowed to ride on their parent atoms with $U_{\text {iso }}(\mathrm{H})=1.2 U_{\text {eq }}(\mathrm{C})$. The highest residual electron density of 1.37 e. $\AA^{-3}$ and the deepest hole of $0.41 \mathrm{e} . \AA^{-3}$ are both located within $1 \AA$ from Se1. Both represent no physical meaning. 


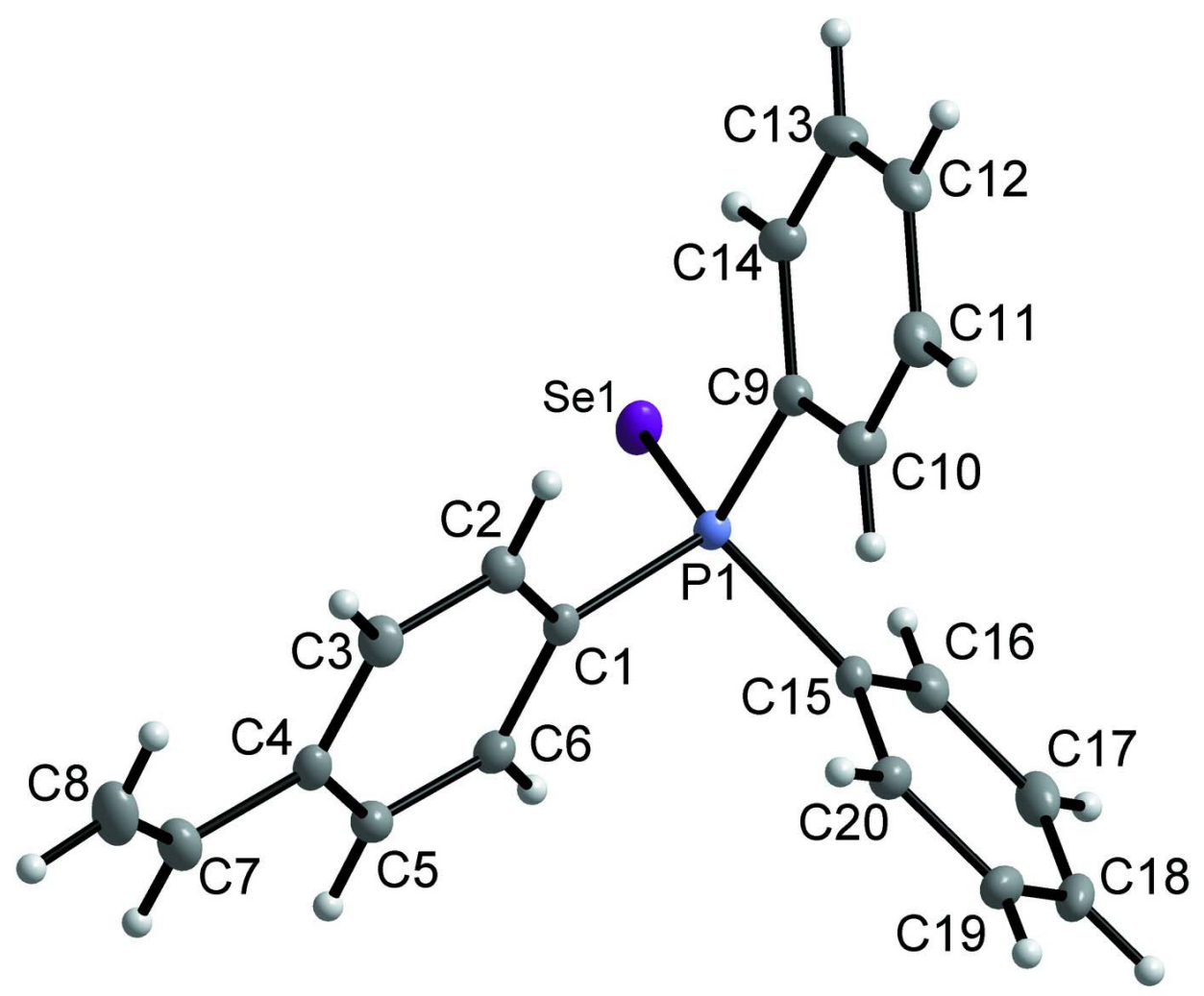

\section{Figure 1}

The molecular structure of the title compound. Displacement ellipsoids are drawn at the $50 \%$ probability level. 


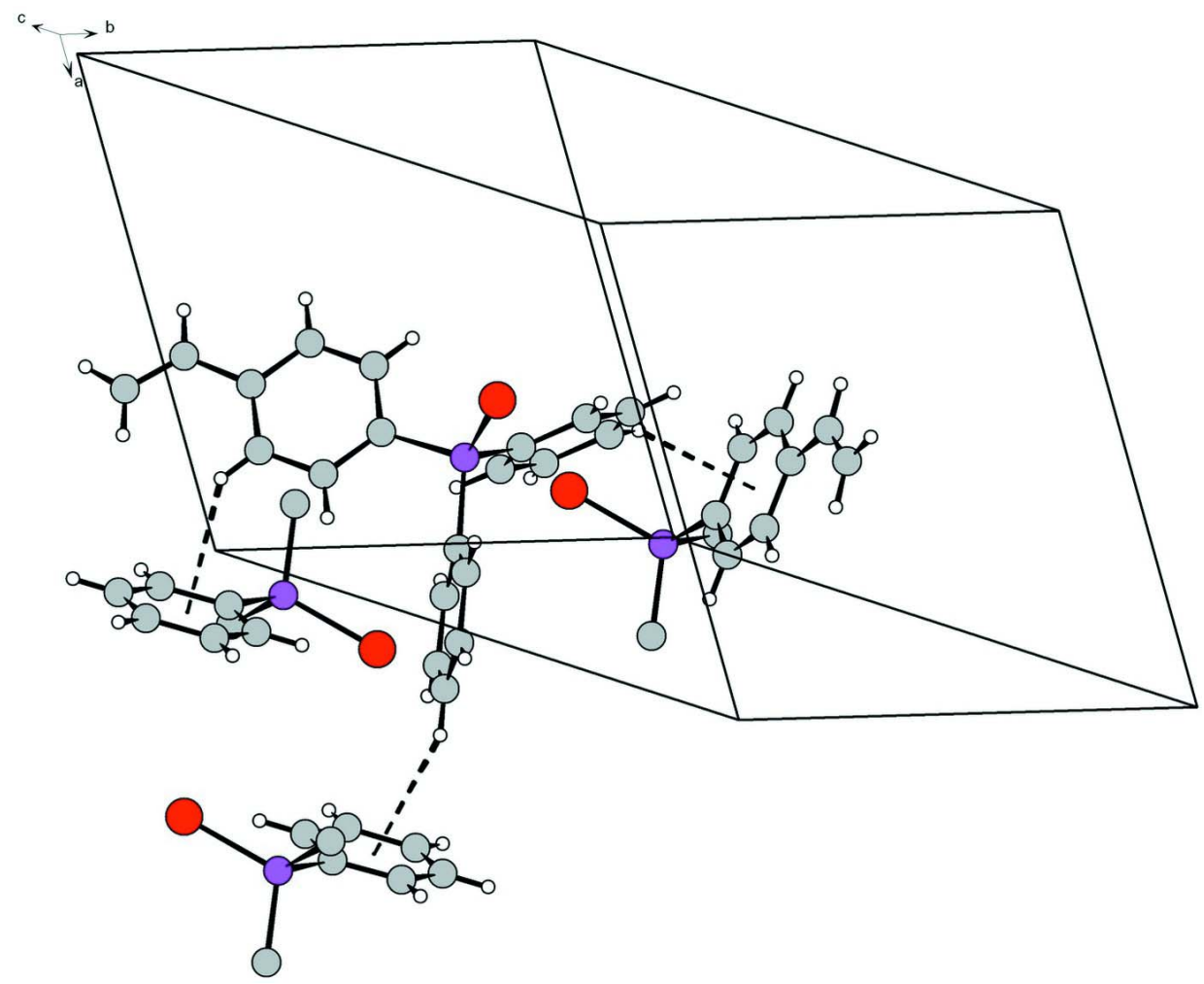

\section{Figure 2}

A packing diagram of the title compound, showing the $\mathrm{C}-\mathrm{H}^{\cdots} \pi \pi$ interactions.

\section{(4-Ethenylphenyl)diphenylphosphine selenide}

Crystal data

$\mathrm{C}_{20} \mathrm{H}_{17} \mathrm{PSe}$

$M_{r}=367.27$

Monoclinic, $P 2_{1} / c$

Hall symbol: -P $2 \mathrm{ybc}$

$a=10.5310(7) \AA$

$b=11.1477(7) \AA$

$c=17.2187(9) \AA$

$\beta=124.562(3)^{\circ}$

$V=1664.66(18) \AA^{3}$

$Z=4$

\section{Data collection}

\section{Bruker APEX DUO 4K-CCD}

diffractometer

Graphite monochromator

Detector resolution: 8.4 pixels $\mathrm{mm}^{-1}$

$\varphi$ and $\omega$ scans

Absorption correction: multi-scan

(SADABS; Bruker, 2008)

$T_{\min }=0.526, T_{\max }=0.737$
$F(000)=744$

$D_{\mathrm{x}}=1.465 \mathrm{Mg} \mathrm{m}^{-3}$

Mo $K \alpha$ radiation, $\lambda=0.71073 \AA$

Cell parameters from 9906 reflections

$\theta=2.5-28.4^{\circ}$

$\mu=2.35 \mathrm{~mm}^{-1}$

$T=100 \mathrm{~K}$

Plate, colourless

$0.3 \times 0.25 \times 0.13 \mathrm{~mm}$

20089 measured reflections

4166 independent reflections

3921 reflections with $I>2 \sigma(I)$

$R_{\text {int }}=0.028$

$\theta_{\text {max }}=28.4^{\circ}, \theta_{\min }=2.3^{\circ}$

$h=-14 \rightarrow 14$

$k=-14 \rightarrow 14$

$l=-16 \rightarrow 22$ 


\section{Refinement}

Refinement on $F^{2}$

Least-squares matrix: full

$R\left[F^{2}>2 \sigma\left(F^{2}\right)\right]=0.024$

$w R\left(F^{2}\right)=0.063$

$S=1.03$

4166 reflections

199 parameters

0 restraints

Primary atom site location: structure-invariant direct methods
Secondary atom site location: difference Fourier map

Hydrogen site location: inferred from neighbouring sites

$\mathrm{H}$-atom parameters constrained

$w=1 /\left[\sigma^{2}\left(F_{\mathrm{o}}^{2}\right)+(0.032 P)^{2}+1.0912 P\right]$ where $P=\left(F_{\mathrm{o}}{ }^{2}+2 F_{\mathrm{c}}{ }^{2}\right) / 3$

$(\Delta / \sigma)_{\max }=0.002$

$\Delta \rho_{\max }=1.37 \mathrm{e}^{-3}$

$\Delta \rho_{\min }=-0.41$ e $\AA^{-3}$

Special details

Experimental. The intensity data was collected on a Bruker Apex DUO 4 K CCD diffractometer using an exposure time of $1.5 \mathrm{~s} /$ frame. A total of 1478 frames were collected with a frame width of $0.5^{\circ}$ covering up to $\theta=28.40^{\circ}$ with $99.9 \%$ completeness accomplished.

Geometry. All e.s.d.'s (except the e.s.d. in the dihedral angle between two 1.s. planes) are estimated using the full covariance matrix. The cell e.s.d.'s are taken into account individually in the estimation of e.s.d.'s in distances, angles and torsion angles; correlations between e.s.d.'s in cell parameters are only used when they are defined by crystal symmetry. An approximate (isotropic) treatment of cell e.s.d.'s is used for estimating e.s.d.'s involving 1.s. planes.

Refinement. Refinement of $F^{2}$ against ALL reflections. The weighted $R$-factor $w R$ and goodness of fit $S$ are based on $F^{2}$, conventional $R$-factors $R$ are based on $F$, with $F$ set to zero for negative $F^{2}$. The threshold expression of $F^{2}>\sigma\left(F^{2}\right)$ is used only for calculating $R$-factors(gt) $e t c$. and is not relevant to the choice of reflections for refinement. $R$-factors based on $F^{2}$ are statistically about twice as large as those based on $F$, and $R$ - factors based on ALL data will be even larger.

Fractional atomic coordinates and isotropic or equivalent isotropic displacement parameters $\left(\hat{A}^{2}\right)$

\begin{tabular}{lllll}
\hline & $x$ & $y$ & $z$ & $U_{\text {iso }} / U_{\text {eq }}$ \\
\hline C1 & $0.32208(15)$ & $0.17035(12)$ & $0.77818(9)$ & $0.0137(2)$ \\
C2 & $0.42929(16)$ & $0.09481(13)$ & $0.85112(10)$ & $0.0168(3)$ \\
H2 & 0.534 & 0.119 & 0.8916 & $0.02^{*}$ \\
C3 & $0.38312(16)$ & $-0.01578(13)$ & $0.86458(10)$ & $0.0179(3)$ \\
H3 & 0.4572 & -0.0668 & 0.9139 & $0.021^{*}$ \\
C4 & $0.22952(17)$ & $-0.05274(12)$ & $0.80657(10)$ & $0.0165(3)$ \\
C5 & $0.12331(16)$ & $0.02363(13)$ & $0.73350(10)$ & $0.0178(3)$ \\
H5 & 0.0186 & -0.0005 & 0.693 & $0.021^{*}$ \\
C6 & $0.16804(16)$ & $0.13364(13)$ & $0.71908(10)$ & $0.0165(3)$ \\
H6 & 0.0942 & 0.1841 & 0.6691 & $0.02^{*}$ \\
C7 & $0.17416(18)$ & $-0.16732(13)$ & $0.81962(11)$ & $0.0222(3)$ \\
H7 & 0.0663 & -0.1817 & 0.7798 & $0.027^{*}$ \\
C8 & $0.2598(2)$ & $-0.25211(14)$ & $0.88126(13)$ & $0.0269(3)$ \\
H8A & 0.3683 & -0.242 & 0.9226 & $0.032^{*}$ \\
H8B & 0.2129 & -0.3232 & 0.8842 & $0.032^{*}$ \\
C9 & $0.58097(15)$ & $0.32505(12)$ & $0.83389(9)$ & $0.0151(2)$ \\
C10 & $0.66759(16)$ & $0.24875(14)$ & $0.81638(10)$ & $0.0198(3)$ \\
H10 & 0.6167 & 0.1951 & 0.7644 & $0.024^{*}$ \\
C11 & $0.82745(17)$ & $0.25109(14)$ & $0.87468(11)$ & $0.0226(3)$ \\
H11 & 0.8857 & 0.1982 & 0.8631 & $0.027^{*}$ \\
C12 & $0.90255(17)$ & $0.33079(15)$ & $0.95012(11)$ & $0.0242(3)$ \\
H12 & 1.012 & 0.3324 & 0.99 & $0.029^{*}$
\end{tabular}




$\begin{array}{lllll}\text { C13 } & 0.81748(18) & 0.40761(15) & 0.96690(11) & 0.0243(3) \\ \text { H13 } & 0.8689 & 0.4629 & 1.0178 & 0.029^{*} \\ \text { C14 } & 0.65719(17) & 0.40446(13) & 0.90988(10) & 0.0195(3) \\ \text { H14 } & 0.5996 & 0.4563 & 0.9227 & 0.023^{*} \\ \text { C15 } & 0.30934(15) & 0.32151(12) & 0.63898(9) & 0.0148(2) \\ \text { C16 } & 0.23401(16) & 0.42233(13) & 0.58296(10) & 0.0189(3) \\ \text { H16 } & 0.2133 & 0.4892 & 0.6084 & 0.023^{*} \\ \text { C17 } & 0.18938(18) & 0.42445(15) & 0.48962(11) & 0.0238(3) \\ \text { H17 } & 0.1383 & 0.4929 & 0.4515 & 0.029^{*} \\ \text { C18 } & 0.21944(18) & 0.32687(15) & 0.45237(11) & 0.0241(3) \\ \text { H18 } & 0.1895 & 0.3289 & 0.3889 & 0.029^{*} \\ \text { C19 } & 0.29308(17) & 0.22632(14) & 0.50744(10) & 0.0216(3) \\ \text { H19 } & 0.3136 & 0.1597 & 0.4817 & 0.026^{*} \\ \text { C20 } & 0.33703(16) & 0.22300(13) & 0.60052(10) & 0.0177(3) \\ \text { H20 } & 0.3859 & 0.1535 & 0.6379 & 0.021^{*} \\ \text { P1 } & 0.37156(4) & 0.31925(3) & 0.76149(2) & 0.01286(8) \\ \text { Se1 } & 0.270418(17) & 0.457326(12) & 0.793367(11) & 0.01969(6) \\ \end{array}$

Atomic displacement parameters $\left(\AA^{2}\right)$

\begin{tabular}{|c|c|c|c|c|c|c|}
\hline & $U^{11}$ & $U^{22}$ & $U^{33}$ & $U^{12}$ & $U^{13}$ & $U^{23}$ \\
\hline $\mathrm{C} 1$ & $0.0185(6)$ & $0.0122(6)$ & $0.0146(6)$ & $-0.0004(5)$ & $0.0119(5)$ & $-0.0007(5)$ \\
\hline $\mathrm{C} 2$ & $0.0169(6)$ & $0.0158(6)$ & $0.0193(6)$ & $0.0008(5)$ & $0.0113(5)$ & 0.0007 (5) \\
\hline $\mathrm{C} 3$ & $0.0205(6)$ & $0.0144(6)$ & $0.0207(7)$ & $0.0033(5)$ & $0.0130(6)$ & $0.0033(5)$ \\
\hline $\mathrm{C} 4$ & $0.0233(7)$ & $0.0135(6)$ & $0.0183(7)$ & $-0.0012(5)$ & $0.0152(6)$ & $-0.0012(5)$ \\
\hline $\mathrm{C} 5$ & $0.0188(6)$ & $0.0190(7)$ & $0.0160(6)$ & $-0.0039(5)$ & $0.0102(5)$ & $-0.0018(5)$ \\
\hline C6 & $0.0195(6)$ & $0.0165(6)$ & $0.0135(6)$ & $-0.0002(5)$ & $0.0094(5)$ & $0.0010(5)$ \\
\hline $\mathrm{C} 7$ & $0.0278(7)$ & $0.0178(7)$ & $0.0266(8)$ & $-0.0043(6)$ & $0.0187(6)$ & $-0.0013(6)$ \\
\hline $\mathrm{C} 8$ & $0.0375(9)$ & $0.0177(7)$ & $0.0359(9)$ & $-0.0010(6)$ & $0.0271(8)$ & $0.0024(6)$ \\
\hline C9 & $0.0162(6)$ & $0.0147(6)$ & $0.0138(6)$ & $-0.0016(5)$ & $0.0082(5)$ & $0.0012(5)$ \\
\hline $\mathrm{C} 10$ & $0.0187(6)$ & $0.0215(7)$ & $0.0192(7)$ & $-0.0013(5)$ & $0.0108(6)$ & $-0.0024(5)$ \\
\hline C11 & $0.0188(7)$ & $0.0267(8)$ & $0.0235(7)$ & $0.0011(6)$ & $0.0127(6)$ & $0.0033(6)$ \\
\hline $\mathrm{C} 12$ & $0.0172(6)$ & $0.0288(8)$ & $0.0204(7)$ & $-0.0038(6)$ & $0.0070(6)$ & $0.0061(6)$ \\
\hline $\mathrm{C} 13$ & $0.0250(7)$ & $0.0234(7)$ & $0.0172(7)$ & $-0.0081(6)$ & $0.0074(6)$ & $-0.0018(6)$ \\
\hline C14 & $0.0236(7)$ & $0.0165(6)$ & $0.0170(7)$ & $-0.0024(5)$ & $0.0107(6)$ & $-0.0003(5)$ \\
\hline $\mathrm{C} 15$ & $0.0155(6)$ & $0.0165(6)$ & $0.0137(6)$ & $-0.0023(5)$ & $0.0091(5)$ & $0.0004(5)$ \\
\hline $\mathrm{C} 16$ & $0.0179(6)$ & $0.0190(7)$ & $0.0194(7)$ & $-0.0011(5)$ & $0.0103(5)$ & $0.0029(5)$ \\
\hline $\mathrm{C} 17$ & $0.0214(7)$ & $0.0273(8)$ & 0.0189 (7) & $-0.0022(6)$ & $0.0093(6)$ & $0.0081(6)$ \\
\hline $\mathrm{C} 18$ & $0.0236(7)$ & $0.0352(9)$ & $0.0140(7)$ & $-0.0090(6)$ & $0.0110(6)$ & $0.0006(6)$ \\
\hline C19 & $0.0241(7)$ & $0.0269(7)$ & $0.0179(7)$ & $-0.0074(6)$ & $0.0145(6)$ & $-0.0060(6)$ \\
\hline $\mathrm{C} 20$ & $0.0197(6)$ & $0.0192(6)$ & $0.0157(6)$ & $-0.0024(5)$ & $0.0108(5)$ & $-0.0014(5)$ \\
\hline P1 & $0.01563(15)$ & $0.01136(15)$ & $0.01348(16)$ & $-0.00006(11)$ & $0.00938(13)$ & $-0.00031(12)$ \\
\hline $\mathrm{Se} 1$ & $0.02669(9)$ & $0.01456(8)$ & $0.02525(9)$ & $0.00329(5)$ & $0.01918(7)$ & $-0.00012(5)$ \\
\hline
\end{tabular}

Geometric parameters $\left(\AA,{ }^{o}\right)$

\begin{tabular}{llll}
\hline $\mathrm{C} 1-\mathrm{C} 2$ & $1.3973(19)$ & $\mathrm{C} 11-\mathrm{C} 12$ & $1.392(2)$ \\
$\mathrm{C} 1-\mathrm{C} 6$ & $1.4005(19)$ & $\mathrm{C} 11-\mathrm{H} 11$ & 0.95
\end{tabular}




\begin{tabular}{|c|c|c|c|}
\hline $\mathrm{C} 1-\mathrm{P} 1$ & $1.8108(14)$ & $\mathrm{C} 12-\mathrm{C} 13$ & $1.383(2)$ \\
\hline $\mathrm{C} 2-\mathrm{C} 3$ & $1.392(2)$ & $\mathrm{C} 12-\mathrm{H} 12$ & 0.95 \\
\hline $\mathrm{C} 2-\mathrm{H} 2$ & 0.95 & $\mathrm{C} 13-\mathrm{C} 14$ & $1.391(2)$ \\
\hline $\mathrm{C} 3-\mathrm{C} 4$ & $1.397(2)$ & C13-H13 & 0.95 \\
\hline $\mathrm{C} 3-\mathrm{H} 3$ & 0.95 & $\mathrm{C} 14-\mathrm{H} 14$ & 0.95 \\
\hline $\mathrm{C} 4-\mathrm{C} 5$ & $1.401(2)$ & $\mathrm{C} 15-\mathrm{C} 20$ & 1.3959 (19) \\
\hline $\mathrm{C} 4-\mathrm{C} 7$ & $1.4733(19)$ & $\mathrm{C} 15-\mathrm{C} 16$ & $1.3981(19)$ \\
\hline $\mathrm{C} 5-\mathrm{C} 6$ & 1.3857 (19) & $\mathrm{C} 15-\mathrm{P} 1$ & $1.8198(14)$ \\
\hline $\mathrm{C} 5-\mathrm{H} 5$ & 0.95 & $\mathrm{C} 16-\mathrm{C} 17$ & $1.396(2)$ \\
\hline $\mathrm{C} 6-\mathrm{H} 6$ & 0.95 & $\mathrm{C} 16-\mathrm{H} 16$ & 0.95 \\
\hline $\mathrm{C} 7-\mathrm{C} 8$ & $1.322(2)$ & $\mathrm{C} 17-\mathrm{C} 18$ & $1.388(2)$ \\
\hline $\mathrm{C} 7-\mathrm{H} 7$ & 0.95 & C17-H17 & 0.95 \\
\hline $\mathrm{C} 8-\mathrm{H} 8 \mathrm{~A}$ & 0.95 & $\mathrm{C} 18-\mathrm{C} 19$ & $1.387(2)$ \\
\hline $\mathrm{C} 8-\mathrm{H} 8 \mathrm{~B}$ & 0.95 & $\mathrm{C} 18-\mathrm{H} 18$ & 0.95 \\
\hline $\mathrm{C} 9-\mathrm{C} 14$ & $1.396(2)$ & $\mathrm{C} 19-\mathrm{C} 20$ & $1.394(2)$ \\
\hline $\mathrm{C} 9-\mathrm{C} 10$ & $1.399(2)$ & C19-H19 & 0.95 \\
\hline $\mathrm{C} 9-\mathrm{P} 1$ & $1.8173(14)$ & $\mathrm{C} 20-\mathrm{H} 20$ & 0.95 \\
\hline $\mathrm{C} 10-\mathrm{C} 11$ & $1.387(2)$ & $\mathrm{P} 1-\mathrm{Se} 1$ & $2.1138(4)$ \\
\hline $\mathrm{C} 10-\mathrm{H} 10$ & 0.95 & & \\
\hline $\mathrm{C} 2-\mathrm{C} 1-\mathrm{C} 6$ & $119.29(12)$ & $\mathrm{C} 13-\mathrm{C} 12-\mathrm{C} 11$ & $119.86(14)$ \\
\hline $\mathrm{C} 2-\mathrm{C} 1-\mathrm{P} 1$ & $122.45(10)$ & $\mathrm{C} 13-\mathrm{C} 12-\mathrm{H} 12$ & 120.1 \\
\hline $\mathrm{C} 6-\mathrm{C} 1-\mathrm{P} 1$ & $118.13(10)$ & $\mathrm{C} 11-\mathrm{C} 12-\mathrm{H} 12$ & 120.1 \\
\hline $\mathrm{C} 3-\mathrm{C} 2-\mathrm{C} 1$ & $120.20(13)$ & $\mathrm{C} 12-\mathrm{C} 13-\mathrm{C} 14$ & $120.49(14)$ \\
\hline $\mathrm{C} 3-\mathrm{C} 2-\mathrm{H} 2$ & 119.9 & $\mathrm{C} 12-\mathrm{C} 13-\mathrm{H} 13$ & 119.8 \\
\hline $\mathrm{C} 1-\mathrm{C} 2-\mathrm{H} 2$ & 119.9 & $\mathrm{C} 14-\mathrm{C} 13-\mathrm{H} 13$ & 119.8 \\
\hline $\mathrm{C} 2-\mathrm{C} 3-\mathrm{C} 4$ & $120.95(13)$ & $\mathrm{C} 13-\mathrm{C} 14-\mathrm{C} 9$ & $120.00(14)$ \\
\hline $\mathrm{C} 2-\mathrm{C} 3-\mathrm{H} 3$ & 119.5 & $\mathrm{C} 13-\mathrm{C} 14-\mathrm{H} 14$ & 120 \\
\hline $\mathrm{C} 4-\mathrm{C} 3-\mathrm{H} 3$ & 119.5 & $\mathrm{C} 9-\mathrm{C} 14-\mathrm{H} 14$ & 120 \\
\hline $\mathrm{C} 3-\mathrm{C} 4-\mathrm{C} 5$ & $118.28(13)$ & $\mathrm{C} 20-\mathrm{C} 15-\mathrm{C} 16$ & $119.64(13)$ \\
\hline $\mathrm{C} 3-\mathrm{C} 4-\mathrm{C} 7$ & $123.00(13)$ & $\mathrm{C} 20-\mathrm{C} 15-\mathrm{P} 1$ & $120.28(10)$ \\
\hline $\mathrm{C} 5-\mathrm{C} 4-\mathrm{C} 7$ & $118.72(13)$ & $\mathrm{C} 16-\mathrm{C} 15-\mathrm{P} 1$ & $120.07(11)$ \\
\hline $\mathrm{C} 6-\mathrm{C} 5-\mathrm{C} 4$ & $121.30(13)$ & $\mathrm{C} 17-\mathrm{C} 16-\mathrm{C} 15$ & $119.78(14)$ \\
\hline $\mathrm{C} 6-\mathrm{C} 5-\mathrm{H} 5$ & 119.4 & $\mathrm{C} 17-\mathrm{C} 16-\mathrm{H} 16$ & 120.1 \\
\hline $\mathrm{C} 4-\mathrm{C} 5-\mathrm{H} 5$ & 119.4 & $\mathrm{C} 15-\mathrm{C} 16-\mathrm{H} 16$ & 120.1 \\
\hline $\mathrm{C} 5-\mathrm{C} 6-\mathrm{C} 1$ & $119.98(13)$ & $\mathrm{C} 18-\mathrm{C} 17-\mathrm{C} 16$ & $120.19(14)$ \\
\hline $\mathrm{C} 5-\mathrm{C} 6-\mathrm{H} 6$ & 120 & $\mathrm{C} 18-\mathrm{C} 17-\mathrm{H} 17$ & 119.9 \\
\hline $\mathrm{C} 1-\mathrm{C} 6-\mathrm{H} 6$ & 120 & $\mathrm{C} 16-\mathrm{C} 17-\mathrm{H} 17$ & 119.9 \\
\hline $\mathrm{C} 8-\mathrm{C} 7-\mathrm{C} 4$ & $126.41(15)$ & $\mathrm{C} 19-\mathrm{C} 18-\mathrm{C} 17$ & $120.23(14)$ \\
\hline $\mathrm{C} 8-\mathrm{C} 7-\mathrm{H} 7$ & 116.8 & $\mathrm{C} 19-\mathrm{C} 18-\mathrm{H} 18$ & 119.9 \\
\hline $\mathrm{C} 4-\mathrm{C} 7-\mathrm{H} 7$ & 116.8 & $\mathrm{C} 17-\mathrm{C} 18-\mathrm{H} 18$ & 119.9 \\
\hline $\mathrm{C} 7-\mathrm{C} 8-\mathrm{H} 8 \mathrm{~A}$ & 120 & $\mathrm{C} 18-\mathrm{C} 19-\mathrm{C} 20$ & $119.99(14)$ \\
\hline $\mathrm{C} 7-\mathrm{C} 8-\mathrm{H} 8 \mathrm{~B}$ & 120 & $\mathrm{C} 18-\mathrm{C} 19-\mathrm{H} 19$ & 120 \\
\hline $\mathrm{H} 8 \mathrm{~A}-\mathrm{C} 8-\mathrm{H} 8 \mathrm{~B}$ & 120 & $\mathrm{C} 20-\mathrm{C} 19-\mathrm{H} 19$ & 120 \\
\hline $\mathrm{C} 14-\mathrm{C} 9-\mathrm{C} 10$ & $119.27(13)$ & $\mathrm{C} 19-\mathrm{C} 20-\mathrm{C} 15$ & $120.14(14)$ \\
\hline $\mathrm{C} 14-\mathrm{C} 9-\mathrm{P} 1$ & $119.62(11)$ & $\mathrm{C} 19-\mathrm{C} 20-\mathrm{H} 20$ & 119.9 \\
\hline $\mathrm{C} 10-\mathrm{C} 9-\mathrm{P} 1$ & $121.08(11)$ & $\mathrm{C} 15-\mathrm{C} 20-\mathrm{H} 20$ & 119.9 \\
\hline $\mathrm{C} 11-\mathrm{C} 10-\mathrm{C} 9$ & $120.29(14)$ & $\mathrm{C} 1-\mathrm{P} 1-\mathrm{C} 9$ & $105.68(6)$ \\
\hline
\end{tabular}




$\begin{array}{llll}\mathrm{C} 11-\mathrm{C} 10-\mathrm{H} 10 & 119.9 & \mathrm{C} 1-\mathrm{P} 1-\mathrm{C} 15 & 104.47(6) \\ \mathrm{C} 9-\mathrm{C} 10-\mathrm{H} 10 & 119.9 & \mathrm{C} 9-\mathrm{P} 1-\mathrm{C} 15 & 107.07(6) \\ \mathrm{C} 10-\mathrm{C} 11-\mathrm{C} 12 & 120.08(14) & \mathrm{C} 1-\mathrm{P} 1-\mathrm{Se} 1 & 113.17(4) \\ \mathrm{C} 10-\mathrm{C} 11-\mathrm{H} 11 & 120 & \mathrm{C} 9-\mathrm{P} 1-\mathrm{Se} 1 & 112.96(5) \\ \mathrm{C} 12-\mathrm{C} 11-\mathrm{H} 11 & 120 & \mathrm{C} 15-\mathrm{P} 1-\mathrm{Se} 1 & 112.82(5) \\ & & & \\ \mathrm{C} 6-\mathrm{C} 1-\mathrm{C} 2-\mathrm{C} 3 & 0.1(2) & \mathrm{C} 16-\mathrm{C} 17-\mathrm{C} 18-\mathrm{C} 19 & 0.4(2) \\ \mathrm{P} 1-\mathrm{C} 1-\mathrm{C} 2-\mathrm{C} 3 & 175.86(11) & \mathrm{C} 17-\mathrm{C} 18-\mathrm{C} 19-\mathrm{C} 20 & 0.1(2) \\ \mathrm{C} 1-\mathrm{C} 2-\mathrm{C} 3-\mathrm{C} 4 & -0.7(2) & \mathrm{C} 18-\mathrm{C} 19-\mathrm{C} 20-\mathrm{C} 15 & -1.0(2) \\ \mathrm{C} 2-\mathrm{C} 3-\mathrm{C} 4-\mathrm{C} 5 & 0.9(2) & \mathrm{C} 16-\mathrm{C} 15-\mathrm{C} 20-\mathrm{C} 19 & 1.5(2) \\ \mathrm{C} 2-\mathrm{C} 3-\mathrm{C} 4-\mathrm{C} 7 & -178.22(14) & \mathrm{P} 1-\mathrm{C} 15-\mathrm{C} 20-\mathrm{C} 19 & -178.21(11) \\ \mathrm{C} 3-\mathrm{C} 4-\mathrm{C} 5-\mathrm{C} 6 & -0.6(2) & \mathrm{C} 2-\mathrm{C} 1-\mathrm{P} 1-\mathrm{C} 9 & 14.26(13) \\ \mathrm{C} 7-\mathrm{C} 4-\mathrm{C} 5-\mathrm{C} 6 & 178.61(13) & \mathrm{C} 6-\mathrm{C} 1-\mathrm{P} 1-\mathrm{C} 9 & -169.95(11) \\ \mathrm{C} 4-\mathrm{C} 5-\mathrm{C} 6-\mathrm{C} 1 & 0.0(2) & \mathrm{C} 2-\mathrm{C} 1-\mathrm{P} 1-\mathrm{C} 15 & 127.04(12) \\ \mathrm{C} 2-\mathrm{C} 1-\mathrm{C} 6-\mathrm{C} 5 & 0.2(2) & \mathrm{C} 6-\mathrm{C} 1-\mathrm{P} 1-\mathrm{C} 15 & -57.17(12) \\ \mathrm{P} 1-\mathrm{C} 1-\mathrm{C} 6-\mathrm{C} 5 & -175.69(11) & \mathrm{C} 2-\mathrm{C} 1-\mathrm{P} 1-\mathrm{Se} 1 & -109.86(11) \\ \mathrm{C} 3-\mathrm{C} 4-\mathrm{C} 7-\mathrm{C} 8 & -4.9(2) & \mathrm{C} 6-\mathrm{C} 1-\mathrm{P} 1-\mathrm{Se} 1 & 65.93(11) \\ \mathrm{C} 5-\mathrm{C} 4-\mathrm{C} 7-\mathrm{C} 8 & 175.95(16) & \mathrm{C} 14-\mathrm{C} 9-\mathrm{P} 1-\mathrm{C} 1 & -115.72(12) \\ \mathrm{C} 14-\mathrm{C} 9-\mathrm{C} 10-\mathrm{C} 11 & 0.8(2) & \mathrm{C} 10-\mathrm{C} 9-\mathrm{P} 1-\mathrm{C} 1 & 62.47(13) \\ \mathrm{P} 1-\mathrm{C} 9-\mathrm{C} 10-\mathrm{C} 11 & -177.41(11) & \mathrm{C} 14-\mathrm{C} 9-\mathrm{P} 1-\mathrm{C} 15 & 133.33(11) \\ \mathrm{C} 9-\mathrm{C} 10-\mathrm{C} 11-\mathrm{C} 12 & -1.0(2) & \mathrm{C} 10-\mathrm{C} 9-\mathrm{P} 1-\mathrm{C} 15 & -48.48(13) \\ \mathrm{C} 10-\mathrm{C} 11-\mathrm{C} 12-\mathrm{C} 13 & 0.0(2) & \mathrm{C} 14-\mathrm{C} 9-\mathrm{P} 1-\mathrm{Se} 1 & 8.54(13) \\ \mathrm{C} 11-\mathrm{C} 12-\mathrm{C} 13-\mathrm{C} 14 & 1.1(2) & \mathrm{C} 10-\mathrm{C} 9-\mathrm{P} 1-\mathrm{Se} 1 & -173.27(10) \\ \mathrm{C} 12-\mathrm{C} 13-\mathrm{C} 14-\mathrm{C} 9 & -1.3(2) & \mathrm{C} 20-\mathrm{C} 15-\mathrm{P} 1-\mathrm{C} 1 & -42.60(12) \\ \mathrm{C} 10-\mathrm{C} 9-\mathrm{C} 14-\mathrm{C} 13 & 0.4(2) & \mathrm{C} 16-\mathrm{C} 15-\mathrm{P} 1-\mathrm{C} 1 & 137.66(11) \\ \mathrm{P} 1-\mathrm{C} 9-\mathrm{C} 14-\mathrm{C} 13 & 178.59(11) & \mathrm{C} 20-\mathrm{C} 15-\mathrm{P} 1-\mathrm{C} 9 & 69.18(12) \\ \mathrm{C} 20-\mathrm{C} 15-\mathrm{C} 16-\mathrm{C} 17 & -1.0(2) & \mathrm{C} 16-\mathrm{C} 15-\mathrm{P} 1-\mathrm{C} 9 & -110.55(11) \\ \mathrm{P} 1-\mathrm{C} 15-\mathrm{C} 16-\mathrm{C} 17 & 178.70(11) & \mathrm{C} 20-\mathrm{C} 15-\mathrm{P} 1-\mathrm{Se} 1 & -165.94(10) \\ \mathrm{C} 15-\mathrm{C} 16-\mathrm{C} 17-\mathrm{C} 18 & 0.1(2) & \mathrm{C} 16-\mathrm{C} 15-\mathrm{P} 1-\mathrm{Se} 1 & 14.33(12) \\ & & & \\ & & & \end{array}$

Hydrogen-bond geometry $\left(\AA,{ }^{\circ}\right)$

$C g 1$ and $C g 2$ are the centroids of the $\mathrm{C} 1-\mathrm{C} 6$ and $\mathrm{C} 15-\mathrm{C} 20$ rings, respectively.

\begin{tabular}{lllll}
\hline$D-\mathrm{H} \cdots A$ & $D-\mathrm{H}$ & $\mathrm{H} \cdots A$ & $D \cdots A$ & $D-\mathrm{H} \cdots A$ \\
\hline $\mathrm{C} 18-\mathrm{H} 18 \cdots C g 1^{\mathrm{i}}$ & 0.95 & 2.62 & $3.383(2)$ & 137 \\
$\mathrm{C} 3-\mathrm{H} 3 \cdots C g 2^{\mathrm{ii}}$ & 0.95 & 2.88 & $3.5889(19)$ & 133 \\
$\mathrm{C} 12-\mathrm{H} 12 \cdots C g 2^{\mathrm{iii}}$ & 0.95 & 2.85 & $3.614(2)$ & 138
\end{tabular}

Symmetry codes: (i) $x,-y-1 / 2, z-3 / 2$; (ii) $-x+1, y-1 / 2,-z+3 / 2$; (iii) $x+1,-y-1 / 2, z-1 / 2$. 